Pacific

Journal of

Mathematics

DUAL PAIRS AND CONTRAGREDIENTS OF IRREDUCIBLE REPRESENTATIONS

BINYONG SUN 


\title{
DUAL PAIRS AND CONTRAGREDIENTS OF IRREDUCIBLE REPRESENTATIONS
}

\author{
BINYONG SUN
}

\begin{abstract}
Let $G$ be one of the classical groups $G L(n), U(n), O(n)$ or $\operatorname{Sp}(2 n)$, over a nonarchimedean local field of characteristic zero. It is well known that the contragredient of an irreducible admissible smooth representation of $G$ is isomorphic to a twist of it by an automorphism of $G$. We prove that similar results hold for double covers of $G$ that occur in the study of local theta correspondences.
\end{abstract}

\section{Introduction and the results}

Fix a nonarchimedean local field $\mathbb{k}$ of characteristic zero. We introduce the notation in order to treat the four classes of classical groups $\mathrm{GL}(n), \mathrm{U}(n), \mathrm{O}(n)$ and $\mathrm{Sp}(2 n)$ simultaneously. Let $A$ be a k-algebra and $\tau$ be a k-algebra involution of $A$ such that

$$
(A, \tau)=\left\{\begin{array}{l}
(\mathbb{k} \times \mathbb{k}, \text { the nontrivial automorphism }), \\
(\text { a quadratic field extension of } \mathbb{k}, \text { the nontrivial automorphism }), \text { or } \\
(\mathbb{k}, \text { the trivial automorphism })
\end{array}\right.
$$

Let $\epsilon= \pm 1$ and let $E$ be an $\epsilon$-Hermitian $A$-module; namely, $E$ is a free $A$-module of finite rank equipped with a nondegenerate $\mathbb{k}$-bilinear map

$$
\langle\cdot, \cdot\rangle_{E}: E \times E \rightarrow A
$$

satisfying $\langle u, v\rangle_{E}=\epsilon\langle v, u\rangle_{E}^{\tau}$ and $\langle a u, v\rangle_{E}=a\langle u, v\rangle_{E}$ for $a \in A$ and $u, v \in E$. Denote by $\mathrm{U}(E)$ the group of all $A$-module automorphisms of $E$ that preserve the form $\langle\cdot, \cdot\rangle_{E}$. Depending on the choice of $A$ and $\epsilon$, it is either a general linear group, a unitary group, an orthogonal group or a symplectic group.

Following Mœglin, Vigneras and Waldspurger [1987, Proposition 4.I.2], we extend $\mathrm{U}(E)$ to a larger group $\breve{\mathrm{U}}(E)$ consisting of pairs $(g, \delta) \in \mathrm{GL}_{\mathbb{k}}(E) \times\{ \pm 1\}$ such that either

$$
\delta=1 \quad \text { and } \quad g \in \mathrm{U}(E),
$$

Supported by NSFC grants number 10801126 and 10931006.

MSC2000: 22E35, 22E46.

Keywords: contragredient representation, dual pair, irreducible representation. 
or

$$
\begin{aligned}
\delta & =-1, \\
g(a u) & =a^{\tau} g(u) \quad \text { for } a \in A, u \in E, \quad \text { and } \\
\langle g u, g v\rangle_{E} & =\langle v, u\rangle_{E} \quad \text { for } u, v \in E .
\end{aligned}
$$

Clearly $\breve{\mathrm{U}}(E)$ contains $\mathrm{U}(E)$ as a subgroup of index two.

In general, if $\pi$ is a representation of a group $H$ and $g$ is an element of a group that acts on $H$ as automorphisms, we define the twist $\pi^{g}$ to be the representation of $H$ that has the same underlying space as that of $\pi$, and whose action is given by $\pi^{g}(h):=\pi(g h)$ for $h \in H$. If $\breve{H}$ is a group containing $H$ as a subgroup of index two, we always let it act on $H$ by conjugation:

$$
\text { Ad }: \breve{H} \times H \rightarrow H, \quad(\breve{g}, x) \mapsto \operatorname{Ad}_{\breve{g}}(x):=\breve{g} x \breve{g}^{-1}
$$

It is a classical result in linear algebra that

$$
\breve{g} x \breve{g}^{-1} \text { is conjugate to } x^{-1} \text { inside } \mathrm{U}(E)
$$

for all $\breve{g} \in \breve{\mathrm{U}}(E) \backslash \mathrm{U}(E)$ and all $x \in \mathrm{U}(E)$. For example, when $\mathrm{U}(E)$ is a general linear group, this amounts to saying that every square matrix is conjugate to its transpose. For orthogonal groups, this says that every element of an orthogonal group is conjugate to its inverse. The following considerations (which lead to Theorem 1.1 below) appear in [Mœglin et al. 1987]. By the localization principle of Bernšteĭn and Zelevinskiǔ [1976, Theorem 6.9 and Theorem 6.15.A], result (1) implies that

$$
f\left(\breve{g} x \breve{g}^{-1}\right)=f\left(x^{-1}\right) \quad(\text { as generalized functions on } \mathrm{U}(E))
$$

for all Ad-invariant generalized functions $f$ on $\mathrm{U}(E)$ and all $\breve{g} \in \breve{\mathrm{U}}(E) \backslash \mathrm{U}(E)$. For the usual notion of generalized functions, see [Sun 2009, Section 2]. We get the following well known result by (2) and by considering characters of irreducible admissible smooth representations (which are conjugation invariant generalized functions).

Theorem 1.1 [Mœglin et al. 1987, Theorem 4.II.1]. Let $\breve{g} \in \breve{\mathrm{U}}(E) \backslash \mathrm{U}(E)$, and let $\pi$ be an irreducible admissible smooth representation of $\mathrm{U}(E)$. Then $\pi^{\vee}$ is isomorphic to $\pi^{\breve{g}}$.

Here and as usual, we use " $\vee "$ to indicate the contragredient of an admissible smooth representation of a totally disconnected locally compact group.

If $E$ is a symplectic space, that is, if $\epsilon=-1$ and $A=\mathbb{k}$, then $\breve{\mathrm{Sp}}(E):=\breve{\mathrm{U}}(E)$ is equal to the subgroup of $\operatorname{GSp}(E)$ with similitudes \pm 1 . Denote by

$$
1 \rightarrow\{ \pm 1\} \rightarrow \tilde{\mathrm{Sp}}(E) \rightarrow \mathrm{Sp}(E) \rightarrow 1
$$


the metaplectic cover of the symplectic group $\mathrm{Sp}(E)$. It is shown in [Mœglin et al. 1987, page 36] that there is a unique continuous action

$$
\widetilde{\mathrm{Ad}}: \breve{\mathrm{Sp}}(E) \times \widetilde{\mathrm{Sp}}(E) \rightarrow \widetilde{\mathrm{Sp}}(E)
$$

of $\breve{\mathrm{Sp}}(E)$ on $\widetilde{\mathrm{Sp}}(E)$ as group automorphisms that lifts the adjoint action

$$
\operatorname{Ad}: \breve{\operatorname{Sp}}(E) \times \operatorname{Sp}(E) \rightarrow \operatorname{Sp}(E)
$$

and leaves the central element $-1 \in \widetilde{\operatorname{Sp}}(E)$ fixed.

We first extend Theorem 1.1 to the case of metaplectic groups:

Theorem 1.2. Assume that $E$ is a symplectic space. Let $\breve{g} \in \breve{\mathrm{Sp}}(E) \backslash \widetilde{\operatorname{Sp}}(E)$, and let $\pi$ be a genuine irreducible admissible smooth representation of $\widetilde{\operatorname{Sp}}(E)$. Then $\pi^{\vee}$ is isomorphic to $\pi^{\breve{g}}$.

Here and henceforth, "genuine" means that the central element $-1 \in \tilde{\operatorname{Sp}}(E)$ acts via the scalar multiplication by -1 .

Remark. In the case that the character of $\pi$ is a locally integrable function, Theorem 1.2 is proved in [Mœglin et al. 1987, Theorem 4.II.2].

Harish-Chandra [1999] proved locally integrability of irreducible characters for $p$-adic linear reductive groups, but he did not treat metaplectic groups.

The proofs of Theorem 1.1 in [Mœglin et al. 1987] and Theorem 1.2 in Section 2 do not depend on locally integrability of irreducible characters.

Now we consider dual pairs. Write $\epsilon^{\prime}:=-\epsilon$, and let $\left(E^{\prime},\langle\cdot, \cdot\rangle_{E^{\prime}}\right)$ be an $\epsilon^{\prime}-$ Hermitian $A$-module. Then $\boldsymbol{E}:=E \otimes_{A} E^{\prime}$ is a skew-Hermitian $A$-module under the form $\left\langle u \otimes u^{\prime}, v \otimes v^{\prime}\right\rangle_{\boldsymbol{E}}:=\langle u, v\rangle_{E}\left\langle u^{\prime}, v^{\prime}\right\rangle_{E^{\prime}}$. Write $\boldsymbol{E}_{\mathbb{k}}:=\boldsymbol{E}$, viewed as a $\mathbb{k}$ symplectic space under the form $\langle u, v\rangle_{\boldsymbol{E}_{\mathbb{k}}}:=\operatorname{tr}_{A / \mathbb{k}}\left(\langle u, v\rangle_{\boldsymbol{E}}\right)$. Put

$$
G:=\mathrm{U}(E), \quad \breve{G}:=\breve{\mathrm{U}}(E), \quad G^{\prime}:=\mathrm{U}\left(E^{\prime}\right), \quad \breve{G}^{\prime}:=\breve{\mathrm{U}}\left(E^{\prime}\right) .
$$

The group $G$ obviously maps to the symplectic group $\operatorname{Sp}\left(\boldsymbol{E}_{\mathbb{k}}\right)$. Define the fiber product $\widetilde{G}:=\tilde{\operatorname{Sp}}\left(\boldsymbol{E}_{\mathbb{k}}\right) \times \operatorname{Sp}\left(\boldsymbol{E}_{\mathbb{k}}\right) G$. This is a double cover of $\mathrm{G}$ that depends on both $E$ and $E^{\prime}$.

In what follows, we define an action

$$
\widetilde{\mathrm{Ad}}: \breve{G} \times \widetilde{G} \rightarrow \widetilde{G}
$$

that lifts the adjoint action Ad: $\breve{G} \times G \rightarrow G$ and fixes the central element $-1 \in \widetilde{G}$. Let $\breve{g}=(g, \delta) \in \breve{G}$. Choose an arbitrary element $\left(g^{\prime}, \delta\right) \in \breve{G}^{\prime}$. Then

$$
\breve{\boldsymbol{g}}:=\left(g \otimes g^{\prime}, \delta\right) \in \breve{\mathrm{Sp}}\left(\boldsymbol{E}_{\mathbb{k}}\right),
$$

and the automorphism

$$
\widetilde{\operatorname{Ad}}_{\breve{g}} \times \operatorname{Ad}_{\breve{g}}: \widetilde{\operatorname{Sp}}\left(\boldsymbol{E}_{\mathbb{k}}\right) \times G \rightarrow \widetilde{\operatorname{Sp}}\left(\boldsymbol{E}_{\mathbb{k}}\right) \times G
$$


leaves the subgroup $\widetilde{G}$ stable. It restricts to an automorphism

$$
\widetilde{\operatorname{Ad}}_{\breve{g}}: \widetilde{G} \rightarrow \widetilde{G}
$$

that is independent of the choice of $g^{\prime}$. We obtain (5) by gluing (7) for all $\breve{g} \in \breve{G}$.

The following is a generalization of Theorem 1.2 in the setting of dual pairs.

Theorem 1.3. Let $\breve{g} \in \breve{G} \backslash G$, and let $\pi$ be a genuine irreducible admissible smooth representation of $\widetilde{G}$. Then $\pi^{\vee}$ is isomorphic to $\pi^{\breve{g}}$.

Remark. When $E^{\prime}=A=\mathbb{k}$ and $\epsilon=-1$, Theorem 1.3 specializes to Theorem 1.2. The statement for the general case reduces essentially to those of Theorem 1.1 and Theorem 1.2. Theorem 1.3 is proved in Section 3.

Theorem 1.3 has the following consequence, which is known to experts (up to a proof of Theorem 1.2). As far as the author knows, no proof of it in full generality is found in the literature.

Theorem 1.4. Denote by $\omega_{\psi}$ the smooth oscillator representation of $\widetilde{\operatorname{Sp}}\left(\boldsymbol{E}_{\mathbb{k}}\right)$ corresponding to a nontrivial character $\psi$ of $\mathbb{k}$. Then for all genuine irreducible admissible smooth representation $\pi$ of $\widetilde{G}$ and $\pi^{\prime}$ of $\widetilde{G}^{\prime}$, we have

$$
\operatorname{dim} \operatorname{Hom}_{G \times G^{\prime}}\left(\omega_{\psi} \otimes \pi \otimes \pi^{\prime}, \mathbb{C}\right)=\operatorname{dim} \operatorname{Hom}_{G \times G^{\prime}}\left(\omega_{\psi}^{\vee} \otimes \pi^{\vee} \otimes \pi^{\prime \vee}, \mathbb{C}\right) .
$$

Here $\widetilde{G}^{\prime}:=\widetilde{\operatorname{Sp}}\left(\boldsymbol{E}_{\mathbb{k}}\right) \times_{\operatorname{Sp}\left(\boldsymbol{E}_{\mathbb{k}}\right)} G^{\prime}$ is a double cover of $G^{\prime}$. Note that both $\omega_{\psi} \otimes \pi \otimes \pi^{\prime}$ and $\omega_{\psi}^{\vee} \otimes \pi^{\vee} \otimes \pi^{\prime \vee}$, which are originally representations of $\widetilde{G} \times \widetilde{G}^{\prime}$, descend to representations of $G \times G^{\prime}$.

Remark. In a follow-up paper [Li et al. 2009], Theorem 1.4 is used to prove multiplicity preservations in theta correspondences (for all residue characteristics), that is, the dimension in Theorem 1.4 is at most one. This is the main reason for providing a detailed proof of Theorem 1.4 here.

In the archimedean case, the analog of Theorem 1.4 is proved by T. Przebinda [1988, Theorem 5.5], while the analog of Theorem 1.3 is a consequence of [1988, Theorem 2.6]. His method is different from ours in that he uses the Langlands classification.

As shown in [Przebinda 1988], Theorem 1.4 together with the Howe duality conjecture implies that theta lifting maps Hermitian representations to Hermitian representations.

\section{Theorem 1.2 and its analog}

Throughout this section, we assume that $\epsilon=-1$. 
2.1. Skew Hermitian modules and Jacobi groups. As in the last section, $E$ is an $\epsilon$-Hermitian $A$-module, and $E_{\mathbb{k}}:=E$ is a symplectic space under the form

$$
\langle u, v\rangle_{E_{\mathbb{k}}}:=\operatorname{tr}_{A / \mathbb{k}}\left(\langle u, v\rangle_{E}\right) .
$$

Denote by $\mathrm{H}(E):=E_{\mathbb{k}} \times \mathbb{k}$ the Heisenberg group associated to $E_{\mathbb{k}}$, whose multiplication is given by $(u, t)\left(u^{\prime}, t^{\prime}\right):=\left(u+u^{\prime}, t+t^{\prime}+\left\langle u, u^{\prime}\right\rangle_{E_{k}}\right)$. The group $\breve{\mathrm{U}}(E)$ acts on $\mathrm{H}(E)$ as group automorphisms by

$$
(g, \delta)(u, t):=(g u, \delta t) .
$$

It defines a semidirect product $\breve{\mathrm{J}}(E):=\breve{\mathrm{U}}(E) \ltimes \mathrm{H}(E)$, which contains $\mathrm{J}(E):=$ $\mathrm{U}(E) \ltimes \mathrm{H}(E)$ as a subgroup of index two.

The results of this note depend heavily on the following.

Lemma 2.1 [Sun 2009, Theorem D]. Let $f$ be a generalized function on $\mathrm{J}(E)$. If $f$ is invariant under conjugations by $\mathrm{U}(E)$, that is,

$$
f\left(g x g^{-1}\right)=f(x) \quad \text { for all } g \in \mathrm{U}(E),
$$

then

$$
f\left(\breve{g} x \breve{g}^{-1}\right)=f\left(x^{-1}\right) \quad \text { for all } \breve{g} \in \breve{\mathrm{U}}(E) \backslash \mathrm{U}(E) .
$$

Actually, we only need the following lemma, which is much weaker.

Lemma 2.2. Let $f$ be a conjugation-invariant generalized function on $\mathrm{J}(E)$. Then

$$
f\left(\breve{g} x \breve{g}^{-1}\right)=f\left(x^{-1}\right) \quad \text { for all } \breve{g} \in \breve{J}(E) \backslash \mathrm{J}(E) .
$$

A consequence of Lemma 2.2 is this:

Proposition 2.3. Let $\breve{g} \in \breve{\mathrm{J}}(E) \backslash \mathrm{J}(E)$, and let $\pi$ be an irreducible admissible smooth representation of $\mathrm{J}(E)$. Then $\pi^{\vee}$ is isomorphic to $\pi^{\breve{g}}$.

Proof. Denote by $f$ the character of $\pi$, which is thus a conjugation-invariant generalized function on $\mathrm{J}(E)$. Therefore

$$
f\left(\breve{g} \times \breve{g}^{-1}\right)=f\left(x^{-1}\right)
$$

by Lemma 2.2. The left side of (9) is the character of $\pi^{\breve{s}}$, and the right side is the character of $\pi^{\vee}$. Therefore $\pi^{\breve{s}}$ and $\pi^{\vee}$ have the same character, and they are thus isomorphic to each other.

2.2. Proof of Theorem 1.2 and its analog. We reuse the notation of Section 2.1. Denote by

$$
\widetilde{\mathrm{U}}(E):=\tilde{\mathrm{Sp}}\left(E_{\mathbb{k}}\right) \times \operatorname{Sp}\left(E_{\mathbb{k}}\right) \mathrm{U}(E)
$$

the double cover of $\mathrm{U}(E)$ induced by the metaplectic cover

$$
1 \rightarrow\{ \pm 1\} \rightarrow \widetilde{\operatorname{Sp}}\left(E_{\mathbb{k}}\right) \rightarrow \operatorname{Sp}\left(E_{\mathbb{k}}\right) \rightarrow 1 .
$$


As in (5), we have an action

$$
\widetilde{\mathrm{Ad}}: \breve{\mathrm{U}}(E) \times \widetilde{\mathrm{U}}(E) \rightarrow \widetilde{\mathrm{U}}(E) .
$$

The following theorem reduces to Theorem 1.2 when $A=\mathbb{k}$.

Theorem 2.4. Assume that $\epsilon=-1$. Let $\breve{g} \in \breve{\mathrm{U}}(E) \backslash \mathrm{U}(E)$, and let $\pi$ be a genuine irreducible admissible smooth representation of $\widetilde{\mathrm{U}}(E)$. Then $\pi^{\vee}$ is isomorphic to $\pi^{\breve{g}}$.

Proof. Denote by $\omega_{\psi}$ the smooth oscillator representation of $\widetilde{\operatorname{Sp}}\left(E_{\mathbb{k}}\right) \ltimes \mathrm{H}(E)$ that corresponds to a nontrivial character $\psi$ of $\mathbb{k}$. Up to isomorphism, this is the only genuine smooth representation that, as a representation of $\mathrm{H}(E)$, is irreducible and has central character $\psi$.

Both $\omega_{\psi}$ and $\pi$ are viewed as smooth representations of $\tilde{\mathbf{J}}(E):=\tilde{\mathrm{U}}(E) \ltimes \mathrm{H}(E)$, via the restriction and the inflation, respectively. The tensor product $\omega_{\psi} \otimes \pi$ descends to an irreducible admissible smooth representation of $\mathrm{J}(E)$ [Sun 2009, Lemma 5.3].

The actions of $\breve{\mathrm{U}}(E)$ on $\widetilde{\mathrm{U}}(E), \mathrm{U}(E)$ and $\mathrm{H}(E)$ induce its actions on the semidirect products $\tilde{\mathbf{J}}(E)$ and $\mathrm{J}(E)$. By Proposition 2.3,

$$
\left(\omega_{\psi} \otimes \pi\right)^{\breve{g}} \cong\left(\omega_{\psi} \otimes \pi\right)^{\vee}
$$

as irreducible admissible smooth representations of $\mathrm{J}(E)$, or equivalently

$$
\omega_{\psi}^{\breve{g}} \otimes \pi^{\breve{g}} \cong \omega_{\psi}^{\vee} \otimes \pi^{\vee}
$$

Note that $\omega_{\psi}^{\breve{g}} \cong \omega_{\psi}^{\vee}$ as smooth representations of $\tilde{\mathbf{J}}(E)$. (This is a special case of Lemma 3.3.) Therefore

$$
\omega_{\psi}^{\vee} \otimes \pi^{\breve{g}} \cong \omega_{\psi}^{\vee} \otimes \pi^{\vee} .
$$

As in the proof of [Sun 2009, Lemma 5.3], we have

$$
\pi^{\breve{g}} \cong \operatorname{Hom}_{\mathrm{H}(E)}\left(\omega_{\psi}^{\vee}, \omega_{\psi}^{\vee} \otimes \pi^{\breve{g}}\right)
$$

Here the right side carries the action of $\widetilde{U}(E)$ given by $(\tilde{g} \phi)(v):=g\left(\phi\left(\tilde{g}^{-1} v\right)\right)$, where

$$
\tilde{g} \in \tilde{\mathrm{U}}(E), \quad \phi \in \operatorname{Hom}_{\mathrm{H}}\left(\omega_{\psi}^{\vee}, \omega_{\psi}^{\vee} \otimes \pi^{\breve{g}}\right), \quad v \in \omega_{\psi}^{\vee},
$$

and $g$ is the image of $\tilde{g}$ under the covering map $\widetilde{U}(E) \rightarrow \mathrm{U}(E)$. Similarly,

$$
\pi^{\vee} \cong \operatorname{Hom}_{H}\left(\omega_{\psi}^{\vee}, \omega_{\psi}^{\vee} \otimes \pi^{\vee}\right) .
$$

We finish the proof by combining (12), (13) and (14). 


\section{Proofs of Theorem 1.3 and Theorem 1.4}

3.1. Proof of Theorem 1.3 for symplectic groups. Now we return to the notation of Section 1. First assume that $A=\mathbb{k}$ and $\epsilon=-1$. Then $G$ is a symplectic group and is thus perfect, that is, $G$ equals its own commutator group. Consequently, there is only one action of $\breve{G}$ on $\widetilde{G}$ that lifts the adjoint action and fixes the central element $-1 \in \widetilde{G}$. There are two cases.

Case 1. The covering map $\widetilde{G} \rightarrow G$ splits. Then $\widetilde{G}=G \times\{ \pm 1\}$, and Theorem 1.3 is one case of Theorem 1.1.

Case 2. The covering map $\widetilde{G} \rightarrow G$ does not split. Then $\widetilde{G}=\widetilde{\operatorname{Sp}}(E)$ [Moore 1968, Theorem 10.4], and Theorem 1.3 is one case of Theorem 1.2.

3.2. Proof of Theorem 1.3 when $A \neq \mathbb{k}$. Assume that $A \neq \mathbb{k}$. Then $\mathrm{U}(\boldsymbol{E})$ is a general linear group or a unitary group.

Lemma 3.1. There exists a genuine character on $\widetilde{\mathrm{U}}(\boldsymbol{E})$.

Proof. It is well known that the exact sequence

$$
1 \rightarrow \mathbb{C}^{\times} \rightarrow\left(\tilde{\mathrm{Sp}}\left(\boldsymbol{E}_{\mathbb{k}}\right) \times \mathbb{C}^{\times}\right) / \operatorname{diag}(\{ \pm 1\}) \rightarrow \operatorname{Sp}\left(\boldsymbol{E}_{\mathbb{k}}\right) \rightarrow 1
$$

splits continuously over $\mathrm{U}(\boldsymbol{E})$ (this is trivial for general linear groups, and for unitary groups, see [Kudla 1994, Proposition 4.1] or [Harris et al. 1996, Section 1]). Write $\iota$ for such a splitting and write $p: \tilde{\mathrm{U}}(\boldsymbol{E}) \rightarrow \mathrm{U}(\boldsymbol{E})$ for the covering map. Then $x \in \widetilde{\mathrm{U}}(\boldsymbol{E}) \mapsto x^{-1} \iota(p(x)) \in \mathbb{C}^{\times}$is a genuine character.

Lemma 3.2. There exists a genuine character $\chi$ of $\widetilde{G}$ such that $\chi^{\breve{g}}=\chi^{-1}$ for all $\breve{g} \in \breve{G} \backslash G$.

Proof. As in Section 1, let $\breve{g}=(g,-1) \in \breve{G} \backslash G$ and $\left(g^{\prime},-1\right) \in \breve{G}^{\prime} \backslash G^{\prime}$, and write $\breve{\boldsymbol{g}}:=\left(g \otimes g^{\prime},-1\right) \in \breve{\mathrm{U}}(\boldsymbol{E}) \backslash \mathrm{U}(\boldsymbol{E})$. It is obvious that the diagram

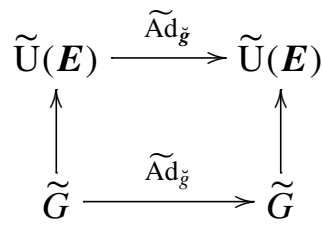

commutes.

Take a character $\chi_{\boldsymbol{E}}$ as in Lemma 3.1, and denote by $\chi$ its restriction to $\widetilde{G}$. Then

$$
\begin{array}{rlrl}
\chi^{\breve{g}} & =\left(\left.\chi_{\boldsymbol{E}}\right|_{\widetilde{G}}\right)^{\breve{g}} & \\
& =\left.\left(\chi_{\boldsymbol{E}}^{\breve{g}}\right)\right|_{\widetilde{G}} & & \text { by commutativity of }(15) \\
& =\left.\left(\chi_{\boldsymbol{E}}^{-1}\right)\right|_{\widetilde{G}} & & \text { by Theorem } 2.4 \\
& =\chi^{-1} . &
\end{array}
$$


Fix $\chi$ as in Lemma 3.2. Let $\breve{g} \in \breve{G} \backslash G$, and let $\pi$ be a genuine irreducible admissible smooth representation of $\widetilde{G}$. Then $\pi \otimes \chi$ descends to an irreducible admissible smooth representation of $G$. By Theorem 1.1, $(\pi \otimes \chi)^{\breve{g}} \cong(\pi \otimes \chi)^{\vee}$, or equivalently, $\pi^{\breve{g}} \otimes \chi^{\breve{g}} \cong \pi^{\vee} \otimes \chi^{-1}$. Therefore, $\pi^{\breve{g}} \cong \pi^{\vee}$ since $\chi^{\breve{g}}=\chi^{-1}$. This proves Theorem 1.3 when $A \neq \mathbb{k}$.

3.3. Proof of Theorem 1.3 for orthogonal groups. Assume that $A=\mathbb{k}$ and $\epsilon=1$, that is, $G$ is an orthogonal group. In what follows, we show that Lemma 3.2 still holds in this case. Fix a complete polarization $E^{\prime}=E_{+}^{\prime} \oplus E_{-}^{\prime}$ of the symplectic space $E^{\prime}$. Then $\boldsymbol{E}=\boldsymbol{E}_{+} \oplus \boldsymbol{E}_{-}$is a complete polarization of the symplectic space $\boldsymbol{E}$, where $\boldsymbol{E}_{ \pm}:=E \otimes E_{ \pm}^{\prime}$. Depending on this polarization, we define a skew-Hermitian $\mathbb{k} \times \mathbb{k}$-module $\boldsymbol{E}^{\prime}$ as follows. As an abelian group, $\boldsymbol{E}^{\prime}=\boldsymbol{E}$. The scalar multiplication is given by

$$
\left(a e_{1}+b e_{2}\right)(u+v):=a u+b v \quad \text { for } a, b \in \mathbb{k}, u \in \boldsymbol{E}_{+}, v \in \boldsymbol{E}_{-},
$$

where $e_{1}:=(1,0)$ and $e_{2}:=(0,1)$ are the two idempotent elements of $\mathbb{k} \times \mathbb{k}$. The skew-Hermitian form is given by

$$
\left\langle u_{+}+u_{-}, v_{+}+v_{-}\right\rangle_{\boldsymbol{E}^{\prime}}:=\left\langle u_{+}, v_{-}\right\rangle_{\boldsymbol{E}} e_{1}+\left\langle u_{-}, v_{+}\right\rangle_{\boldsymbol{E}} e_{2},
$$

where $u_{+}, v_{+} \in \boldsymbol{E}_{+}, u_{-}, v_{-} \in \boldsymbol{E}_{-}$.

Let $\breve{g}=(g,-1) \in \breve{G} \backslash G$. Choose an element $\left(g^{\prime},-1\right) \in \breve{G}^{\prime} \backslash G^{\prime}$ such that $g^{\prime}\left(E_{+}^{\prime}\right)=E_{-}^{\prime}$ and $g^{\prime}\left(E_{-}^{\prime}\right)=E_{+}^{\prime}$. Then

$$
\breve{\boldsymbol{g}}:=\left(g \otimes g^{\prime},-1\right) \in \breve{\mathrm{U}}\left(\boldsymbol{E}^{\prime}\right) \backslash \mathrm{U}\left(\boldsymbol{E}^{\prime}\right),
$$

and we have a commutative diagram

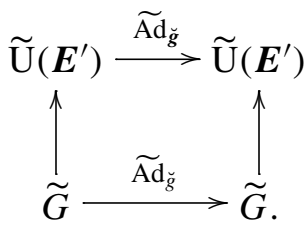

Take a genuine character $\chi_{\boldsymbol{E}^{\prime}}$ of $\widetilde{\mathrm{U}}\left(\boldsymbol{E}^{\prime}\right)$ as in Lemma 3.1, and denote by $\chi$ its restriction to $\widetilde{G}$. Then as in the proof of Lemma 3.2, we show that $\chi$ fulfills the requirement of Lemma 3.2. Now we argue as in the end of the last subsection, and prove Theorem 1.3 for orthogonal groups.

3.4. Proof of Theorem 1.4. The group

$$
\breve{G}:=\breve{G} \times_{\{ \pm 1\}} \breve{G}^{\prime}=\left\{\left(g, g^{\prime}, \delta\right) \mid(g, \delta) \in \breve{G},\left(g^{\prime}, \delta\right) \in \breve{G}^{\prime}\right\}
$$

contains $\boldsymbol{G}:=G \times G^{\prime}$ as a subgroup of index two. Define a homomorphism

$$
\xi: \breve{\boldsymbol{G}} \rightarrow \breve{\operatorname{Sp}}\left(\boldsymbol{E}_{\mathbb{k}}\right), \quad\left(g, g^{\prime}, \delta\right) \mapsto\left(g \otimes g^{\prime}, \delta\right) .
$$


By using the covering map $\widetilde{G} \times \widetilde{G}^{\prime} \rightarrow \boldsymbol{G}=G \times G^{\prime}$ and the map $\left.\xi\right|_{\boldsymbol{G}}: \boldsymbol{G} \rightarrow \operatorname{Sp}\left(\boldsymbol{E}_{\mathbb{k}}\right)$, we form the semidirect product $\left(\widetilde{G} \times \widetilde{G}^{\prime}\right) \ltimes \mathrm{H}(\boldsymbol{E})$ as in Section 2.1. Let $\breve{G}$ act on $\left(\widetilde{G} \times \widetilde{G}^{\prime}\right) \ltimes \mathrm{H}(\boldsymbol{E})$ as group automorphisms by

$$
\breve{\boldsymbol{g}}(x, y, z):=\left(\widetilde{\operatorname{Ad}}_{\breve{g}}(x), \widetilde{\operatorname{Ad}}_{\breve{g}^{\prime}}(y), \xi(\breve{\boldsymbol{g}}) z\right),
$$

where

$$
\breve{\boldsymbol{g}}=\left(g, g^{\prime}, \delta\right), \quad \breve{g}=(g, \delta), \quad \breve{g}^{\prime}=\left(g^{\prime}, \delta\right),
$$

and the last term of the right hand side of (16) is defined as in (8).

Let $\omega_{\psi}, \pi$ and $\pi^{\prime}$ be as in Theorem 1.4.

Lemma 3.3. View $\omega_{\psi}$ as an admissible smooth representation of $\left(\widetilde{G} \times \widetilde{G}^{\prime}\right) \ltimes \mathrm{H}(\boldsymbol{E})$ (via the restriction). Then for every $\breve{\boldsymbol{g}} \in \breve{\boldsymbol{G}} \backslash \boldsymbol{G}$, we have

$$
\omega_{\psi}^{\vee} \cong \omega_{\psi}^{\breve{g}} .
$$

Proof. Recall that the group $\breve{\mathrm{Sp}}\left(\boldsymbol{E}_{\mathbb{k}}\right)$ acts on $\widetilde{\operatorname{Sp}}\left(\boldsymbol{E}_{\mathbb{k}}\right) \ltimes \mathrm{H}(\boldsymbol{E})$ diagonally through its action on the two factors. We have

$$
\omega_{\psi}^{\vee} \cong \omega_{\psi}^{\xi(\breve{g})}
$$

as smooth oscillator representations of $\widetilde{\mathrm{Sp}}\left(\boldsymbol{E}_{\mathbb{k}}\right) \ltimes \mathrm{H}(\boldsymbol{E})$, since both correspond to the character $\psi^{-1}$. We prove the lemma by restricting both sides of (17) to the group $\left(\widetilde{G} \times \widetilde{G}^{\prime}\right) \ltimes \mathrm{H}(\boldsymbol{E})$.

Lemma 3.4. Via the inflations, view $\pi$ and $\pi^{\prime}$ as admissible smooth representations of $\left(\widetilde{\boldsymbol{G}} \times \widetilde{\boldsymbol{G}}^{\prime}\right) \ltimes \mathrm{H}(\boldsymbol{E})$. Then for every $\breve{\boldsymbol{g}} \in \breve{\boldsymbol{G}} \backslash \boldsymbol{G}$, we have

$$
\pi^{\vee} \cong \pi^{\breve{g}} \text { and } \pi^{\prime \vee} \cong \pi^{\prime \breve{g}} \text {. }
$$

Proof. Write $\breve{g}=\left(g, g^{\prime},-1\right)$ and $\breve{g}=(g,-1)$. By Theorem 1.3, we have $\pi^{\vee} \cong \pi^{\breve{g}}$ as irreducible admissible smooth representations of $\widetilde{G}$. By pulling back this isomorphism to the group $\left(\widetilde{G} \times \widetilde{G}^{\prime}\right) \ltimes \mathrm{H}(\boldsymbol{E})$, we obtain the first isomorphism of (18). The second isomorphism follows similarly.

Lemma 3.5. For every $\breve{\boldsymbol{g}} \in \breve{\boldsymbol{G}} \backslash \boldsymbol{G}$, we have

$$
\omega_{\psi}^{\vee} \otimes \pi^{\vee} \otimes \pi^{\prime \vee} \cong\left(\omega_{\psi} \otimes \pi \otimes \pi^{\prime}\right)^{\breve{g}}
$$

as smooth representations of $\left(\widetilde{G} \times \widetilde{G}^{\prime}\right) \ltimes \mathrm{H}(\boldsymbol{E})$.

Proof. This is a combination of Lemma 3.3 and Lemma 3.4.

Fix an element $\breve{\boldsymbol{g}} \in \breve{\boldsymbol{G}} \backslash \boldsymbol{G}$. Since the action of $\breve{\boldsymbol{g}}$ stabilizes the subgroup $\widetilde{G} \times \widetilde{G}^{\prime}$ of $\left(\widetilde{G} \times \widetilde{G}^{\prime}\right) \ltimes \mathrm{H}(\boldsymbol{E})$, we have

$$
\operatorname{Hom}_{\widetilde{G} \times \widetilde{G}^{\prime}}\left(\omega_{\psi} \otimes \pi \otimes \pi^{\prime}, \mathbb{C}\right)=\operatorname{Hom}_{\widetilde{G} \times \widetilde{G}^{\prime}}\left(\left(\omega_{\psi} \otimes \pi \otimes \pi^{\prime}\right)^{\breve{g}}, \mathbb{C}\right) .
$$

Now Theorem 1.4 is a consequence of (19) and (20). 


\section{Acknowledgments}

I thank the referee for many detailed suggestions.

\section{References}

[Bernšteĭn and Zelevinskiı̌ 1976] I. N. Bernšter̆n and A. V. Zelevinskǐ̌, "Representations of the group GL $(n, F)$, where $F$ is a local non-Archimedean field", Uspehi Mat. Nauk 31:3 (1976), 5-70. In Russian; translated in Russian Mathematical Surveys 31:3 (1976), 1-68. MR 54 \#12988

[Harish-Chandra 1999] Harish-Chandra, Admissible invariant distributions on reductive $p$-adic groups, University Lecture Series 16, American Mathematical Society, Providence, RI, 1999. MR 2001b:22015 Zbl 0928.22017

[Harris et al. 1996] M. Harris, S. S. Kudla, and W. J. Sweet, "Theta dichotomy for unitary groups", J. Amer. Math. Soc. 9:4 (1996), 941-1004. MR 96m:11041 Zbl 0870.11026

[Kudla 1994] S. S. Kudla, "Splitting metaplectic covers of dual reductive pairs", Israel J. Math. 87:1-3 (1994), 361-401. MR 95h:22019 Zbl 0840.22029

[Li et al. 2009] J.-S. Li, B. Sun, and Y. Tian, "The multiplicity one conjecture for local theta correspondences", preprint, 2009. To appear in Invent. Math. arXiv 0903.1419

[Mœglin et al. 1987] C. Mœglin, M.-F. Vignéras, and J.-L. Waldspurger, Correspondances de Howe sur un corps p-adique, Lecture Notes in Mathematics 1291, Springer, Berlin, 1987. MR 91f:11040 Zbl 0642.22002

[Moore 1968] C. C. Moore, "Group extensions of p-adic and adelic linear groups", Inst. Hautes Études Sci. Publ. Math. 35 (1968), 157-222. MR 39 \#5575 Zbl 0159.03203

[Przebinda 1988] T. Przebinda, “On Howe's duality theorem”, J. Funct. Anal. 81:1 (1988), 160-183. MR 89j:22031 Zbl 0678.22007

[Sun 2009] B. Sun, "Multiplicity one theorems for Fourier-Jacobi models", preprint, 2009. arXiv 0903.1417

Received December 7, 2009. Revised April 21, 2010.

BINYONG SUN

ACADEMY OF MATHEMATICS AND SyStems SCIENCE

CHINESE ACADEMY OF SCIENCES

BEIJING 100190

CHINA

sun@math.ac.cn 


\title{
PACIFIC JOURNAL OF MATHEMATICS
}

\author{
http://www.pjmath.org \\ Founded in 1951 by
}

E. F. Beckenbach (1906-1982) and F. Wolf (1904-1989)

\section{EDITORS}

V. S. Varadarajan (Managing Editor)

Department of Mathematics

University of California

Los Angeles, CA 90095-1555

pacific@math.ucla.edu

Vyjayanthi Chari

Department of Mathematics

University of California

Riverside, CA 92521-0135

chari@math.ucr.edu

\section{Robert Finn}

Department of Mathematics Stanford University

Stanford, CA 94305-2125

finn@math.stanford.edu

Kefeng Liu

Department of Mathematics

University of California

Los Angeles, CA 90095-1555

liu@math.ucla.edu
Darren Long

Department of Mathematics

University of California

Santa Barbara, CA 93106-3080

long@math.ucsb.edu

Jiang-Hua Lu

Department of Mathematics

The University of Hong Kong

Pokfulam Rd., Hong Kong jhlu@maths.hku.hk

Alexander Merkurjev

Department of Mathematics University of California

Los Angeles, CA 90095-1555 merkurev@math.ucla.edu
Sorin Popa

Department of Mathematics

University of California

Los Angeles, CA 90095-1555

popa@math.ucla.edu

Jie Qing

Department of Mathematics

University of California

Santa Cruz, CA 95064

qing@ cats.ucsc.edu

Jonathan Rogawski

Department of Mathematics

University of California

Los Angeles, CA 90095-1555

jonr@math.ucla.edu

\section{PRODUCTION}

pacific@math.berkeley.edu

Silvio Levy, Scientific Editor Matthew Cargo, Senior Production Editor

ACADEMIA SINICA, TAIPEI

CALIFORNIA INST. OF TECHNOLOGY

INST. DE MATEMÁTICA PURA E APLICADA

KEIO UNIVERSITY

MATH. SCIENCES RESEARCH INSTITUTE

NEW MEXICO STATE UNIV.

OREGON STATE UNIV.

\section{SUPPORTING INSTITUTIONS}

STANFORD UNIVERSITY
UNIV. OF BRITISH COLUMBIA
UNIV. OF CALIFORNIA, BERKELEY
UNIV. OF CALIFORNIA, DAVIS
UNIV. OF CALIFORNIA, LOS ANGELES
UNIV. OF CALIFORNIA, RIVERSIDE
UNIV. OF CALIFORNIA, SAN DIEGO
UNIV. OF CALIF., SANTA BARBARA

UNIV. OF CALIF., SANTA CRUZ

UNIV. OF MONTANA

UNIV. OF OREGON

UNIV. OF SOUTHERN CALIFORNIA

UNIV. OF UTAH

UNIV. OF WASHINGTON

WASHINGTON STATE UNIVERSITY

These supporting institutions contribute to the cost of publication of this Journal, but they are not owners or publishers and have no responsibility for its contents or policies.

See inside back cover or www.pjmath.org for submission instructions.

The subscription price for 2011 is US \$420/year for the electronic version, and \$485/year for print and electronic.

Subscriptions, requests for back issues from the last three years and changes of subscribers address should be sent to Pacific Journal of Mathematics, P.O. Box 4163, Berkeley, CA 94704-0163, U.S.A. Prior back issues are obtainable from Periodicals Service Company, 11 Main Street, Germantown, NY 12526-5635. The Pacific Journal of Mathematics is indexed by Mathematical Reviews, Zentralblatt MATH, PASCAL CNRS Index, Referativnyi Zhurnal, Current Mathematical Publications and the Science Citation Index.

The Pacific Journal of Mathematics (ISSN 0030-8730) at the University of California, c/o Department of Mathematics, 969 Evans Hall, Berkeley, CA 94720-3840, is published monthly except July and August. Periodical rate postage paid at Berkeley, CA 94704, and additional mailing offices. POSTMASTER: send address changes to Pacific Journal of Mathematics, P.O. Box 4163, Berkeley, CA 94704-0163.

PJM peer review and production are managed by EditFLOW ${ }^{\mathrm{TM}}$ from Mathematical Sciences Publishers.

PUBLISHED BY PACIFIC JOURNAL OF MATHEMATICS

at the University of California, Berkeley 94720-3840

A NON-PROFIT CORPORATION

Typeset in IATEX

Copyright $(2011$ by Pacific Journal of Mathematics 


\section{PACIFIC JOURNAL OF MATHEMATICS}

Volume $249 \quad$ No. $2 \quad$ February 2011

A gluing construction for prescribed mean curvature

257

ADRIAN BUTSCHER

Large eigenvalues and concentration

271

BRUNO COLBOIS and ALESSANDRO SAVO

Sur les conditions d'existence des faisceaux semi-stables sur les courbes multiples primitives

JEAN-MARC DRÉZET

A quantitative estimate for quasiintegral points in orbits

LIANG-CHUNG HSIA and JosePh H. Silverman

Möbius isoparametric hypersurfaces with three distinct principal curvatures, II

ZEJUN HU and SHUJIE ZHAI

Discrete Morse theory and Hopf bundles

371

DMITRY N. KOZLOV

Regularity of canonical and deficiency modules for monomial ideals

ManOJ KUMmini and SATOSHI MURAI

$\mathrm{SL}_{2}(\mathbb{C})$-character variety of a hyperbolic link and regulator

WEIPING LI and QINGXUE WANG

Hypergeometric evaluation identities and supercongruences

LING LONG

Necessary and sufficient conditions for unit graphs to be Hamiltonian

H. R. Maimani, M. R. Pournaki and S. Yassemi

Instability of the geodesic flow for the energy functional

DOMENico PERrone

String structures and canonical 3-forms

CORBETT REDDEN

Dual pairs and contragredients of irreducible representations

BINYONG SUN

On the number of pairs of positive integers $x_{1}, x_{2} \leq H$ such that $x_{1} x_{2}$ is a $k$-th power

DOYCHIN I. TOLEV

Correction to the article A Floer homology for exact contact embeddings 